\section{Translational challenges from the 2014 Gastrointestinal Cancers Symposium: toward a true tailored therapy through effective research}

\author{
Giuseppe Aprile', Francesco Giuliani², Stefano Cordio ${ }^{3}$, Andrea Sartore-Bianchi ${ }^{4}$, \\ Katia Bencardino ${ }^{4}$, Elena Ongaro', Concetta Martines ${ }^{3}$, Riccardo Giampieri ${ }^{5}$, Roberto \\ Bordonaro $^{3}$, Salvatore Siena ${ }^{4}$, Stefano Cascinu ${ }^{5} \&$ Mario Scartozzi*,5 $^{*}$
}

\section{Gastrointestinal Cancers Symposium 2014, San Francisco, CA, USA, 16-18 January 2014}

The Gastrointestinal Cancers Symposium represents an indisputable occasion for sharing results and research opportunities for investigators around the globe. Across the years along with clinical trials presentations the meeting increasingly acquired a distinct role as a scientific arena for translational research. Also, this year the need for predictive markers for first-generation targeted agents and research about novel biologically driven therapeutic options characterized most of the studies presented. We focus here on reports from the 2014 American Society of Clinical Oncology (ASCO) Gastrointestinal Cancers Symposium indicating an opportunity for biological selection of either the pharmacological target or the patient population in order to enhance clinical outcome.

\section{Metastatic colorectal cancer as a paradigm for molecularly tailored therapy: from bench to bedside \& back \\ At the 2014 American Society of Clinical Oncology (ASCO) Gastrointestinal Cancers Symposium new data have been presented, integrating previous knowledge and further findings supporting extended $R A S$ molecular selection for EGFR-targeted therapeutics. Marc Peeters presented the prospective ret- rospective analysis of mutations in KRAS exons 3 and 4 and $N R A S$ exons 2, 3 and 4 in patients with known wild-type (WT) KRAS exon 2 mutations from the Phase III study 181 of panitumumab plus FOLFIRI (irinotecan, 5-FU and leucovorin) versus FOLFIRI in second-line [1], showing 18\% addi- tional RAS mutations among WT KRAS exon 2 patients and that treatment effect of panitumumab plus FOLFIRI vs FOLFIRI was enhanced for overall survival (OS; hazard ratio [HR]: 0.85-0.80) and progression-free survival (PFS; HR: 0.73-0.69) in the WT RAS group versus the WT KRAS exon 2-limited group. Furthermore, several data of front-line cetuximab-based regimens according to $R A S$ status have been presented. Sebastian Stintzing and colleagues reported a preplanned analysis of the FIRE-3 study of FOLFIRI plus cetuximab versus FOLFIRI plus bevacizumab as first-line treat- ment, covering expanded $R A S$ mutations and mutations of BRAF, PIK3CA (exon 9 and 20) and $A k t$ by pyrosequencing [2]. Besides confirming the remarkable gain of 7.5 months in OS in the RAS WT population in favor of FOLFIRI plus cetuximab (HR: 0.70; $\mathrm{p}=0.011$ ) and the negative prognostic}

'Medical Oncology, University \& General Hospital, Udine, Italy

${ }^{2}$ Medical Oncology, IRCCS Ospedale Oncologico di Bari, Bari, Italy

${ }^{3}$ Medical Oncology, Ospedale Garibaldi Catania, Catania, Italy

${ }^{4}$ Niguarda Cancer Center, Ospedale Niguarda Ca' Granda, Milano, Italy

${ }^{5}$ Medical Oncology Department, Translational Research Unit Ospedali Riuniti di Ancona, Università Politecnica delle Marche, Ancona, Italy

*Author for correspondence: Tel.: +39 0715963834; Fax: +39 0715964192; marioscartozzi@gmail.com

\section{KEYWORDS}

- colorectal cancer

- gastrointestinal

cancers symposium 2014

- hepatocellular carcinoma

- pancreatic cancer

- translational research 
role of $B R A F$ mutations, these authors showed the novel finding that PIK3CA mutations (additional $7.3 \%$ of patient population) were associated with a clear trend for PFS favoring FOLFIRI plus bevacizumab (median PFS: 13.3 vs 7.8 months) and higher response rate (RR; 57.9 vs $47.4 \%$ ). These data, although not reaching statistical significance, might warrant further investigations as PIK3CA mutations have been already proposed as negative biomarkers to cetuximab in metastatic colorectal cancer [3,4]. Finally, Sabine Tejpar presented treatment outcomes of the OPUS study of cetuximab plus FOLFOX-4 (oxaliplatin, 5-FU and leucovorin) according to additional KRAS exons 3 and 4 (4.5\%) and NRAS exons 2, 3 and $4(10 \%)$ using BEAMing, showing that any activating mutation of $R A S$ precludes benefit from the addition of cetuximab [5]. Altogether, these findings enhance data from previous analyses and demonstrate that appropriate molecular selection is mandatory for achieving maximum benefit, possibly allowing also recognition and understanding of the best choice between antiangiogenic and EGFR-targeted treatment in first-line of metastatic colorectal cancer.

In a different field of investigation, Nishi Kothari and colleagues presented data from Moffitt Cancer Center (FL, USA) and Royal Melbourne Hospital (Australia) to explore the association between regular aspirin use and survival in metastatic colorectal cancer, showing that in patients harboring a $P I K 3 C A$ mutation ( $\mathrm{n}=187$ ) regular aspirin use was associated with a significant overall (HR: $0.35 ; \mathrm{p}=0.04$ ) and cancer-specific (HR: 0.28 ; $\mathrm{p}=0.02$ ) survival advantage [6]. Interestingly, this study, which is the largest to examine aspirin use in PIK3CAmutated colorectal cancer, did not confirm the survival advantage in earlier stages (II-III) of the disease, thus posing a challenge to previous knowledge.

\section{Developing biologically driven treatment strategies in esophageal \& gastric cancers}

A multimodal treatment encompassing chemoradiation followed by radical surgery is considered the standard of care for localized esophageal cancers worldwide. Since approximately $25 \%$ of patients do not respond to preoperative chemoradiation, a join team of investigators from M.D. Anderson Cancer Center (TX, USA) and Castle Biosciences (TX, USA) developed the DecisionDx-EC test, a three-protein biomarker assay able to predict with a proprietary algorithm which patients are likely to be resistant to the upfront induction treatment. An initial study of 167 patients demonstrated an accuracy of 92\% and a specificity of $97 \%$. In the current validation cohort study [7], immunohistochemical staining for $\mathrm{NF}-\kappa \mathrm{B}$, Gli1 and SHH proteins were performed on samples obtained from 71 patients and a labeling index score was assigned. The negative predictive value of $83 \%$ and the specificity of $94 \%$ indicate that this test could accurately identify patients not likely to benefit from induction therapy (those with post-treatment tumor shrinkage $<50 \%$ ). Resistant patients might therefore avoid toxic and ineffective chemoradiation, and may be assigned to alternative preoperative regimens or directly proceed to radical surgery.

The importance of epithelial-mesenchimal transition (EMT) has been recognized as a potential cause for cancer progression in different gastrointestinal malignancies. A novel genetically engineered mouse model was developed mating conditional knockout mice of Smad4, p53 and E-cadherin with $P d x-1$-Cre transgenic mice [8]. The recently presented model confirmed that loss of E-cadherin and Smad4 cooperate with p53 loss to promote the growth and progression of stomach adenocarcinoma, histologically similar to human diffuse gastric cancer, and suggests that EMT may become a potential therapeutic target.

Despite the negative results of bevacizumab in the first-line setting, the introduction of ramucirumab contributed to regain interest in angiogenic inhibitors in gastric cancer. RAINBOW, a randomized Phase III trial, compared paclitaxel plus ramucirumab, a human IgG1 antibody against VEGFR-2, to paclitaxel alone in 665 pretreated advanced gastric cancer patients [9]. The combination resulted superior in median OS (9.6 vs 7.3 months; HR: 0.8), median PFS (4.4 vs 2.8 months; HR: 0.63$)$ and RR (28 vs $16 \% ; \mathrm{p}<0.001)$. Hypertension, fatigue and neutropenia were more frequently reported in patients exposed to ramucirumab, but the incidence of febrile neutropenia was comparable between arms.

HCC patients: biological \& clinical heterogeneity as potential treatment keys In the EVOLVE-1 study, 546 patients with BCLC stage B or C HCC, whose disease progressed during or after sorafenib or who were sorafenib intolerant, were randomized 2:1 to receive everolimus at $7.5 \mathrm{mg}$ daily plus best supportive care or placebo plus best supportive care [10]. At the final analysis 
no difference in median OS was observed between experimental and placebo arms. Also, median time to progression was similar, whereas the disease control rate was better in the everolimus arm (56.1 vs $45.1 \%$; $\mathrm{p}=0.01$ ).

In a further trial trebananib, a drug that sequesters Ang-1 and Ang-2, preventing their interaction with the Tie- 2 receptor, was added to sorafenib in first-line Child-Pugh A HCC patients [11]. Two cohorts, each of 30 patients, were treated with trebananib administered intravenously at $10 \mathrm{mg} / \mathrm{kg}$ weekly (cohort 1 ) or at $15 \mathrm{mg} / \mathrm{kg}$ weekly (cohort 2) while sorafenib was given at $400 \mathrm{mg} /$ twice daily. The study did not meet the primary end point of $62 \%$ PFS at 4 months. Also, inhibition of the IGF-1 pathway was tested. In a Phase I study the monoclonal antibody cixutumumab was employed in combination with sorafenib [12]. Grade 3 diarrhea (19\%), hypertension (19\%), thrombocytopenia (14\%), palmar-plantar erythrodysthestesia (10\%), hyperglicemia (10\%) and fatigue $(10 \%)$ were the main observed toxicityies. Among the 16 evaluable patients, 13 obtained stable disease with a median event-free survival and $O S$ of 3.8 and 13.1 months, respectively. The TGF- $\beta 1$ receptor 1 kinase inhibitor Ly 2157299 monohydrate was tested in HCC patients pretreated with or ineligible to sorafenib [13]. A total fo 109 patients were included. In the overall population a median time to progression (TTP) of 12 weeks was observed (12.1/10.0 weeks). In the sorafenib-naive group, the median TTP was 18.3 weeks. TTP was higher in the nonalcohol compared with alcohol-only etiology group (12.1 vs 6.1). The median OS (overall survival) was 36 weeks and was better in AFP responders than in nonresponders. Globally it is evident that the challenge in the next few years will be to categorize the different types of HCC by molecular signature and to identify predictive biomarkers of response and resistance [14].

\section{Into the dark side of pancreatic tumors: new insights, new options}

Heestand et al. presented updated data from the RTOG 9704 trial on the multiplex capabilities and high-sensitivity detection of the proximity ligation assay (PLA), a probe panel capable of quantifying $42 \mathrm{key}$ proteins from patient serum [15]. This trial confirmed the prognostic value of CA 19.9 and CEA level, while a low level of (MMP-7 predicted OS benefit, thus confirming PLA as powerful tool to identify biomarkers from a small volume of serum samples.
Researchers from University of California, Los Angeles (UCLA; CA, USA) presented data about diagnostic and prognostic value of pancreatic circulating tumor cells [16]. The authors' findings suggest that circulating tumor cell presence can aid the diagnosis of pancreatic cancer both in terms of separation of locally advanced and metastatic disease with high specificity.

K-RAS mutational status and its biological role were investigated in different patients' series [17-19]. In a retrospective series from the Wayne State University of Detroit (MI, USA) the frequency of patients with WT K-RAS tumors was higher than previously described. These patients were also biologically distinct from those with mutated $K R A S$. Moreover, findings from an Italian patient series indicated that oncogenic KRAS is directly associated with SHH expression and that the Hedgehog pathway components may be relevant therapeutic targets for KRAS-mutant patients.

Finally, researchers from the San Louis University (MO, USA) presented a retrospective analysis of patients with pancreatic cancer using next-generation sequencing. They described common mutations such as KRAS and TP53, but also revealed genomic alterations that may be potentially responsive to available targeted agents such as novel mutations in WT KRAS tumors, which warrant further investigation.

In the research for biomarkers of gemcitabine resistance, tumor samples from patients randomized to gemcitabine or FOLFOX (oxaliplatin, 5-FU and leucovorin) were prospectively tested for hENT1 expression [20]. The study supports the need for further research into the predictive value of hENT1.

\section{Conclusion}

The introduction of targeted agents for the treatment of solid tumors made the need of predictive biomarkers increasingly relevant. Along with this fast-growing area of research it also became evident that prospective clinical trials testing novel agents should assume a different design incorporating as many molecular suggestions from translational studies as possible.

In the treatment of metastatic colorectal tumors the validation of the extended $R A S$ analysis for anti-EGFR treatment is a clear example of this exciting field of research [21]. This process is now also ongoing in gastric, pancreatic and hepatocellular tumors, and findings presented at the Gastrointestinal Cancers Symposium 2014 
are an indication of the progress we are making. We still have a long journey ahead, but the road now looks safer than it previously did.

\section{Financial \& competing interests disclosure}

The authors have no relevant affiliations or financial involvement with any organization or entity with a financial interest in or financial conflict with the subject matter or materials discussed in the manuscript. This includes employment, consultancies, honoraria, stock ownership or options, expert testimony, grants or patents received or pending, or royalties.

No writing assistance was utilized in the production of this manuscript.

\section{References}

1 Peeters M, Oliner KS, Price TJ et al. Analysis of KRAS/NRAS mutations in Phase 3 study 20050181 of panitumumab (pmab) plus FOLFIRI versus FOLFIRI for second-line treatment (tx) of metastatic colorectal cancer (mCRC). J. Clin. Oncol. 32 (Suppl. 3), Abstract LBA387 (2014).

2 Stintzing S, Jung A, Rossius L et al. Mutations within the EGFR signaling pathway: Influence on efficacy in FIRE-3 - a randomized Phase III study of FOLFIRI plus cetuximab or bevacizumab as first-line treatment for wild-type (WT) KRAS (exon 2) metastatic colorectal cancer (mCRC) patients. J. Clin. Oncol. 32(Suppl. 3), Abstract 445 (2014).

3 Sartore-Bianchi A, Martini M, Molinari F et al. PIK3CA mutations in colorectal cancer are associated with clinical resistance to EGFR-targeted monoclonal antibodies. Cancer Res. 69, 1851-1857 (2009).

4 Sartore-Bianchi A, Di Nicolantonio F, Nichelatti $\mathrm{M}$ et al. Multi-determinants analysis of molecular alterations for predicting clinical benefit to EGFR-targeted monoclonal antibodies in colorectal cancer. PLoS ONE 4(10), e7287 (2009).

5 Tejpar S, Lenz HJ, Köhne CH et al. Effect of KRAS and NRAS mutations on treatment outcomes in patients with metastatic colorectal cancer (mCRC) treated first-line with cetuximab plus FOLFOX4: new results from the OPUS study. J. Clin.

Oncol. 32(Suppl. 3), Abstract LBA444 (2014).

6 Kothari N, Kim RD, Gibbs P et al. Regular aspirin (ASA) use and survival in patients with PIK3CA-mutated metastatic colorectal cancer (CRC). J. Clin. Oncol. 32 (Suppl. 3), Abstract 386 (2014).

7 Shan Wl, Lassen N, Johnson C et al. A propietary multianalyte test for predicting extreme resistance to neoadjuvant 5 -FU based chemoradiation (CTRT) in esophageal adenocar- cinoma (EC). Presented at: 2014 Gastrointestinal Cancers Symposium. San Francisco, CA, USA, 16-18 January 2014.
8 Kim HK, Park JW, Green JE. Relationship of novel genetically engineered mouse and the epithelial-mesenchymal transition in the metastastic progression of gastric cancers. Presented at: 2014 Gastrointestinal Cancers Symposium. San Francisco, CA, USA, 16-18 January 2014.

9 Wilke H, Van Cutsem E, Oh SC et al. RAINBOW: a global, Phase III, randomized, double-blind study of ramuci- rumab plus paclitaxel versus placebo plus paclitaxel in the treatment of metastatic gastroesophageal junction (GEJ) and gastric adenocarcinoma following disease progression on first-line platinum- and fluoropyrimidine- containing combination therapy rainbow IMCL CP12-0922 (I4T-IE- JVBE). Presented at: 2014 Gastrointestinal Cancers Symposium. San Francisco, CA, USA, 16-18 January 2014.

10 Zhu AX, Kudo M, Assenat E et al. EVOLVE-1: Phase III study of everolimus for advanced HCC that progressed during or after sorafenib. Presented at: 2014 Gastrointestinal Cancers Symposium. San Francisco, CA, USA, 16-18 January 2014.

11 Abou-Alfa GK, Blanc J, Miles $S$ et al. Phase II study of first-line trebananib plus sorafenib in patients with advanced hepatocellular carcinoma (HCC). Presented at: 2014 Gastrointestinal Cancers Symposium. San Francisco, CA, USA, 16-18 January 2014.

12 El-Khoueiry AB, O'Donnell R, Mack PC et al. A Phase I trial of cixutumumab (C) (IMC-A12) and sorafenib (S) for treatment of advanced hepatocellular carcinoma (HCC). Presented at: 2014 Gastrointestinal Cancers Symposium. San Francisco, CA, USA, 16-18 January 2014.

13 Faivre SJ, Santoro A, Kalley RK et al. A Phase II study of a novel transforming growth factor-beta (TGF-beta1) receptor 1 kinase inhibitor, Ly2157299 monohydrate (Ly), in patients with advanced hepatocellular carcinoma (HCC). Presented at: 2014 Gastrointestinal Cancers Symposium. San Francisco, CA, USA, 16-18 January 2014.

14 Faloppi L, Scartozzi M, Maccaroni E et al. Evolving strategies for the treatment of hepatocellular carcinoma: from clinicalguided to molecularly-tailored therapeutic options. Cancer Treat. Rev. 37, 169-177 (2011).

15 Heestand GM, Murphy DM, Moughan J et al. A novel biomarker panel examining response to adjuvant pancreatic cancer therapy in RTOG 9704. Presented at: 2014 Gastrointestinal Cancers Symposium. San Francisco, CA, USA, 16-18 January 2014.

16 Ankeny JS, Hou S, Lin M et al. Pancreatic circulating tumour cells as a diagnostic adjunct in pancreatic cancer. Presented at: 2014 Gastrointestinal Cancers Symposium. San Francisco, CA, USA, 16-18 January 2014.

17 Salem ME, Al-Hajeili MR, Dyson G et al. Clinicopathologic features of patients with KRAS wild-type pancreatic adenocarcinoma. Presented at: 2014 Gastrointestinal Cancers Symposium. San Francisco, CA, USA, 16-18 January 2014.

18 Santoni M, Bittoni A, Andrikou K et al. Correlation of KRAS mutational state with the expression of the hedgehog pathway in patients with pancreatic ductal adenocarcinoma. Presented at: 2014 Gastrointestinal Cancers Symposium. San Francisco, CA, USA, 16-18 January 2014.

19 Teague AS, Tan BR, Lockhart AC et al. Next-generation sequencing in pancreatic cancer: revealing genomic mutations beyond KRAS. Presented at: 2014 Gastrointestinal Cancers Symposium. San Francisco, CA, USA, 16-18 January 2014.

20 Chua YJ, Karapetis CS, Gebski V et al. Human equilibrative nucleoside transporter 1 (hENT1) in gemcitabine and FOLFOX (oxaliplatin, 5-fluorouracil, and leucovorin)treated patients with metastatic pancreatic cancer: the randomized Phase II PAN1 study. Presented at: 2014 Gastrointestinal Cancers Symposium. San Francisco, CA, USA, 16-18 January 2014

21 Giampieri R, Scartozzi M, Del Prete M et al. Molecular biomarkers of resistance to anti-EGFR treatment in metastatic colorectal cancer, from classical to innovation. Crit. Rev. Oncol. Hematol. 88, 272-283 (2013). 